\title{
L'indépendance menacée de la presse médicale
}

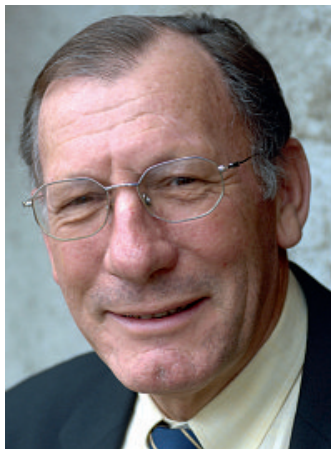

Jean Martin

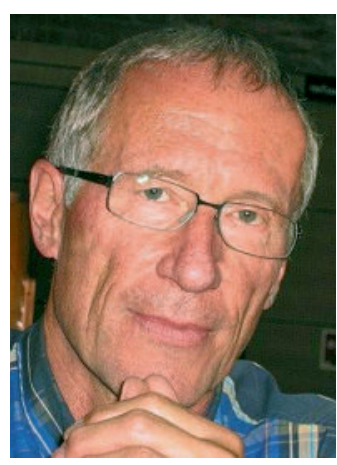

Erhard Taverna
Nous sommes dans un monde où la communication joue un rôle déterminant. On vient de le vivre avec le «printemps arabe» ou avec la réaction américaine contre Osama ben Laden. Il y a cinquante ans, Marshall McLuhan nous surprenait avec la formule «Le médium est le message», soulignant qu'il n'y pas que la substance qui compte mais bien aussi la manière de transmettre.

Les médecins doivent être partie prenante des décisions prises dans la Cité à propos de leur mission, de leur vocation - (presque) tout est res publica, vie de la polis donc politique. Qu'on les aime ou pas, les lobbyistes sont parmi nous, au moins 300 à Berne, 15000 à Bruxelles, plus encore à Washington. Même si un certain lobbying doit susciter de sérieuses réserves parce que le pouvoir de l'argent y est excessif (à la limite de la corruption), défendre ses intérêts est un droit, c'est légitime, nécessaire. On n'imagine pas aujourd'hui un groupe d'intérêts ou une profession qui ne disposerait pas d'organes adéquats d'information.

Ce billet entend dire du bien d'une presse médicale indépendante. Pour défendre les intérêts des médecins comme ceux de la population et en son sein tout spécialement de ceux qui sont malades, blessés, handicapés - et qui, dans notre ordre constitutionnel ont le droit de recevoir de soins de qualité et bien accessibles. Pour œuvrer dans ce but, nous devons communiquer adéquatement.

Pendant longtemps les anciens (ceux de notre âge), dans un monde plus «familial», ont vécu avec des journaux médicaux largement subventionnés par l'industrie pharmaceutique, comme tant de réunions de formation continue. Mais la problématique des conflits d'intérêts, dont on parlait peu ou pas alors, est devenue à juste titre un enjeu majeur. En médecine comme ailleurs, on s'est rendu compte que même des personnes raisonnables et qui ont des principes tendaient à se montrer aimables vis-à-vis de ceux qui leur font des cadeaux. D'où les mesures prises pour limiter le potentiel d'influence sur la pratique des médecins et sur ce qu'ils lisent dans leurs heures de formation. Ainsi, l'article 33 de la loi sur les produits thérapeutiques interdit de promettre ou d'accepter des avantages matériels. Comme pour l'industrie du médicament et des appareils, il importe que des contrepoids suffisants soient apportés, au plan de la société, aux influences excessives d'autres secteurs, notamment celui de l'assurance-maladie (les caisses ont compris l'importance de communiquer tous azimuts et de faire pression, mettant de leur côté beaucoup de gens d'influence).

Si on ajoute à cela les effets de la crise qui a secoué le monde depuis 2008, dont une forte baisse des montants que l'industrie attribue à la publicité, on a l'explication des soucis financiers généralisés de la presse écrite médicale. Occasion de se souvenir de «We do not have problems, we only have opportunities»: ces problèmes sont la chance de renforcer notre engagement concret pour l'indépendance des revues professionnelles et scientifiques; à nous de démontrer que nous voulons mettre à distance les conflits d'intérêts vis-àvis d'autres secteurs - qui sont des partenaires obligés au sein du système de santé mais dont les objectifs ne sont pas les mêmes que ceux des soignants que nous sommes.

Dans une étude de "Transparency International» de 2010, la Suisse tenait un très bon $8^{\mathrm{e}}$ rang, sur 180 pays, en ce qui concerne la liberté de la presse. Le fait que la presse ne se laisse pas intimider par de possibles boycotts publicitaires y était un critère important. Une branche professionnelle comme la nôtre, dont les revenus restent de bon niveau, doit maintenir la crédibilité de ses publications en assurant l'essentiel de leur financement. La liberté, cela coûte quelque chose.

Un mot sur les mutations qu'entraînent les supports électroniques dans toute la sphère de la communication. Les éditeurs "papier», qui pour certains sont issus d'une tradition remontant à Gutenberg, comme Schwabe notre éditeur, sont conscients de la nécessité d'évoluer; internet et d'autres moyens sont de plus en plus utilisés pour les transmissions de tous ordres - ainsi au plan de la science, de la formation continue et de la politique professionnelle. Toutefois, pour quelques décennies encore, on peut parier que se maintiendra le plaisir de tenir un journal ou un livre entre les mains, de s'asseoir confortablement pour parcourir un document imprimé. Nos enfants auront leur manière de faire, mais pour l'instant maintenons à un haut niveau de qualité les instruments qui nous servent bien.

En résumé, il s'agit d'être conscients des enjeux liés à l'existence et à la prospérité (à la santé!) d'une presse médicale indépendante, pertinente, et qui nous donne du plaisir par la qualité et la diversité de ce qu'elle apporte. Qui, entre autres choses, est un trait d'union entre nous et une carte de visite de la profession.

Jean Martin et Erhard Taverna* ancien médecin cantonal d'Appenzell RhodesExtérieures. 